\title{
Imaging and Documenting Gammarideans
}

\author{
Carolin Haug, Gerd Mayer, Verena Kutschera, Dieter Waloszek, \\ Andreas Maas, and Joachim T. Haug
}

Workgroup Biosystematic Documentation, University of Ulm, Helmholtzstraße 20, $89081 \mathrm{Ulm}$, Germany

Correspondence should be addressed to Carolin Haug, carolin_haug@gmx.de

Received 29 March 2011; Revised 30 May 2011; Accepted 30 May 2011

Academic Editor: Almut Gerhardt

Copyright ( 2011 Carolin Haug et al. This is an open access article distributed under the Creative Commons Attribution License, which permits unrestricted use, distribution, and reproduction in any medium, provided the original work is properly cited.

\begin{abstract}
We give an overview of available techniques for imaging and documenting applied to gammarideans and discuss their advantages and disadvantages. Although recent techniques, such as confocal laser scanning microscopy (cLSM), focused ion beam scanning electron microscopy (FIB SEM), or computed microtomography ( $\mu \mathrm{CT}$ ), provide new possibilities to detect and document structures, these high-tech devices are expensive, and access to them is often limited. Alternatively, there are many possibilities to enhance the capabilities of established techniques such as macrophotography and light microscopy. We discuss improvements of the illumination with polarized light and the possibilities of utilizing the autofluorescence of animals such as the gammarideans. In addition, we present software-based enhancing tools such as image fusion and image stitching.
\end{abstract}

\section{Introduction}

Imaging and documenting specimens is an important part of the basic biological investigations, particularly of morphological and taxonomic work. Informative images are also required for oral or poster presentations. In recent years, new documentation techniques for zoomorphological investigations have been developed. Studying the morphology appears to have become an increasingly "high-tech" field of science, demanding complex machines and fast computers with complicated software packages. Despite this, new ideas involving facile and inexpensive methods and free computer software capable of running on older computers have become available and can improve classical morphological approaches significantly. Notable among these new high-tech methods are tools for three-dimensional documentation, such as different types of computed tomography (CT) (e.g., [1]) and confocal laser scanning microscopy (cLSM) (e.g., $[2,3])$. The new tools for enhancing more classical methods are mainly based on computer algorithms, such as image fusion or image stitching (e.g., $[4,5]$ ), in addition to $3 \mathrm{D}$ approaches such as structure from motion (e.g., [6, 7]). Furthermore, the combination of different methods and also the adoption of techniques from one field into another have yielded promising results.
New techniques can also offer new insights. Former complex preparation processes that were necessary to answer certain questions may become superfluous (at least in some cases). They have been superseded by new methods yielding comparable results which may be faster and/or do not require the destruction of rare specimens. Examples of such cases are computer tomography substituting serial sectioning [8] and fluorescence microscopy being used instead of alizarin staining [9].

The crustacean taxon Gammaridea with almost 6,000 living species must be considered a well-investigated group, at least taxonomically. Despite this, many morphological details of the numerous described species remain significantly understudied, and many structures are still waiting to be discovered and understood (e.g., $[10,11]$ ). This is despite such detailed morphological studies being the basis for investigations of ecological, phylogeographical, or evolutionary aspects of the species being studied. Conclusions about the ecology of these animals, for example, on the ecological interplay of native and neozoic animals, are tightly coupled to the understanding of the detailed morphology of the species in focus $[12,13]$.

For the investigations of morphological aspects of gammarideans, newly emerged techniques yield new opportunities. Therefore, we tested a large variety of up-to-date 
documentary techniques, compared them against one another, and considered the advantages and disadvantages of each technique with a special focus on the cost-benefit equation. Tested techniques ranged from computer microtomography to different types of fluorescence microscopy to enhanced variants of white-light microscopy and macrophotography. We aimed at offering an overview of available techniques applicable to facilitate and improve future studies on gammarideans.

\section{Different Methods and Discussion}

2.1. General Remarks. Specimens of the following gammaridean species were the basis of the present investigation: Gammarus roeselii Gervais, 1835, Dikerogammarus villosus Sowinsky, 1894, Dikerogammarus haemobaphes (Eichwald, 1841), and Orchestia cavimana Heller, 1865. In general different types of software were used to optimize the recorded images. To overcome limitations of depth of field of an image, several images of the same image detail were recorded in different focus levels. These images form a socalled image stack, with one single image referred to as "frame". Frames of a stack were fused with freely available image fusion software (CombineZM, CombineZP, Image Analyzer) to one sharp image. To overcome limitations of field of view, several images were combined to one panorama with image stitching software (Adobe Photoshop CS3 "Photomerge" function or the freely available Microsoft Image Composite Editor). Adobe Photoshop CS3 or Gimp was also used to optimize contrast and brightness. In slightly blurred images, "mask unsharp" filters were used to improve these.

\subsection{Documenting Entire Specimens with Macrophotography}

2.2.1. Methods. A live specimen of Gammarus roeselii was photographed with a Panasonic FZ-50 digital camera, equipped with an additional Raynox M-150 macroscopic lens. Two synchronized Nikon SB-20 flashlights were used for illumination.

Complete female specimens of Dikerogammarus villosus, which had been stored in $70 \%$ alcohol, were documented with macrophotography in their storage liquid. They were placed in front of black velvet background and photographed with a Canon macrophoto lens EF-S $60 \mathrm{~mm}$ on a Canon EOS $450 \mathrm{D}$ digital camera as a stack of frames under different settings. (1) Under "normal” light. Two Leica KL 1500 coldlight sources were used to illuminate the specimen, with the angle of light at about $45^{\circ}$ to minimize reflections. (2) Under crossed polarized light [14]. A polarizing filter was not only mounted on the camera lens but also on two cold-light sources. Filters on the two cold-light sources were adjusted parallel to each other, and the filter on the camera lens was adjusted with perpendicular filter direction. (3) Fluorescence settings [9]. Cold-light sources were equipped with cyan filters and the camera lens with a red filter. (4) A stereo image under polarized light. In the settings $1-3$, stacks were recorded and fused (see above).

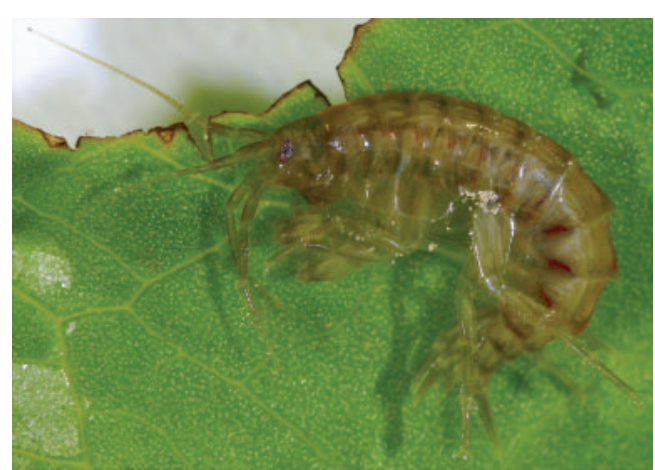

FIGURE 1: Macrophotographic image of a living gammaridean, Gammarus roeselii. An additional Raynox M-150 macroscopic lens was mounted on a Panasonic FZ-50 digital camera. Illumination with two synchronized Nikon SB-20 flashlights.

2.2.2. Documenting Gammarideans Alive or Dead. vDue to the fast movements of their limbs, especially of the continuously beating pleopods, the entire living gammarideans can only be documented by a single image, best illuminated using a flashlight (Figure 1). The advantage of such images is the retention of the natural color and appearance of the specimen on an image and the possibility to depict the specimen in its natural surrounding. However, such images usually suffer from two difficulties. First, often not all structures of the specimen are entirely sharp, because the depth of field is limited. The second difficulty is that reflections often occur.

With dead or anesthetized specimens, the first problem can be overcome by recording several frames of the same image detail in different focal layers and by fusing the resulting stack of frames by the application of a computer software for image fusion into one sharp image of high depth of field. Such programs are freely downloadable from the internet today and are usually easy to handle. With a camera equipped with a macro lens, it is often sufficient to take two or three frames in a stack to obtain a sharp final image. Image fusion programs also compensate size or shift differences while refocusing, usually occurring when using macro lenses and also stereo microscopes. More problematic are rotations, but even this issue can be overcome if recognized later. Yet if it is recognized right after recording, we recommend just a rerecording of the stack, because this is often less time-consuming and more accurate. For macrophotography, the camera should be mounted on a tripod or a repro stand. With the latter, it is possible to change the distance between the lens and the object in small steps, without rotating or moving the camera in the other two dimensions.

For illumination, flashlights or cold-light lamps can be used. With cold-light lamps, it is possible to adjust the camera and control settings in live preview. Many modern digital cameras are supplied with remote software so that it is possible to control the camera directly on the computer and view a live preview on the screen.

2.2.3. Getting Rid of Reflections: Polarized Light. An important shortcoming of photographing stored specimens is 


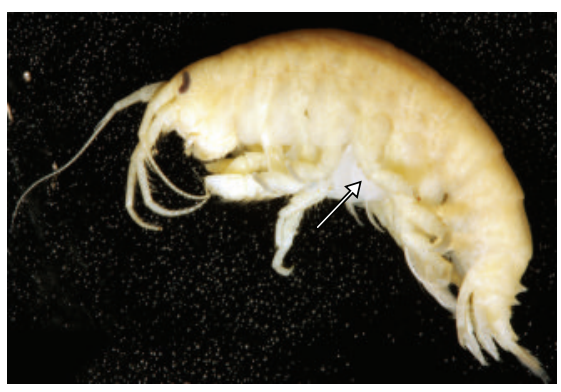

(a)

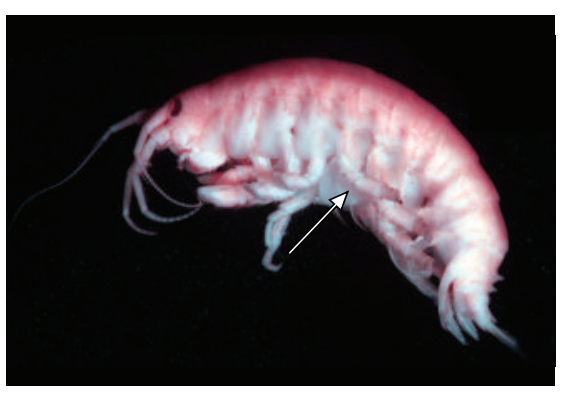

(c)

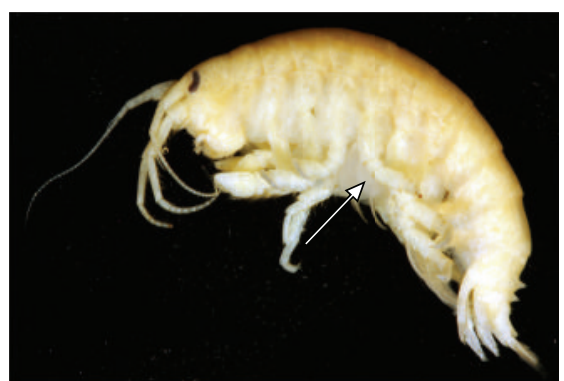

(b)

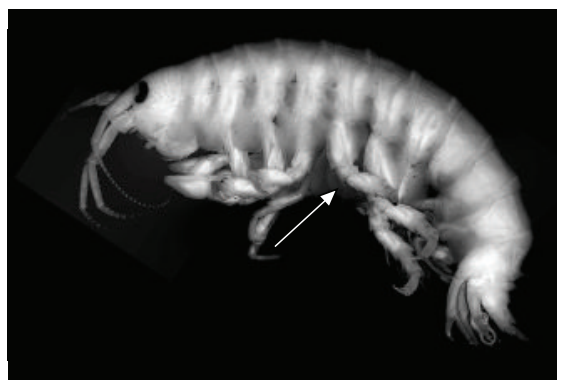

(d)

FIgURE 2: Comparison of different methods applied to an entire specimen of Dikerogammarus villosus stored in alcohol. All images are fused from several focal planes. (a)-(c) Macrographs. (a) "Normal" macrograph, with optimized uniform light. Bubbles are evident on the black velvet, which was used as background. Due to reflections, individual elements of the limbs are difficult to distinguish (arrow). (b) Macrograph under polarized light. Bubbles are not apparent. Borders between individual elements of the limbs more conspicuous (arrow). (c) Macrofluorescence. Borders between individual limb elements are well apparent due to the color contrast (arrow). (d) Composite fluorescence image [4]. Details well contrasted, also the individual limb elements (arrow).

reflections caused by the storage liquid. Even if the specimen is removed from its liquid, the wet surface produces a lot of reflections. Taking the specimen out may also cause the damage of the specimen as it may desiccate; at least, setae may easily agglutinate. Photographing wet specimens "wet-dry" is especially not recommended for rare material. Photographing the specimens immersed in a sufficient amount of their storage liquid moves the surface reflection away from the specimen, although this usually does not completely erase all reflections (Figure 2(a)).

An easy solution to get rid of reflections is the application of crossed polarizing filters. Polarizing filter foils are cheap and can be easily attached to the light sources. One after the other is then turned until maximum elimination of reflections is achieved. This can be best tested by adjusting the filters while observing a piece of polished metal, for example, pincers or needles. When all filters on the light sources are adjusted parallel, the filter on the camera lens is turned until maximum elimination of reflections is achieved, adjusted with perpendicular filter direction. When using black velvet as a background polarizing filters make especially the small bubbles invisible that usually cover at least in some areas of the submersed velvet (cf. Figure 2(a) versus 2(b)). For the best results and undisturbed metering, the filter mounted on the camera lens should not be a piece of polarizing filter foil, but a commercial circular polarizing filter.

Applying polarized light has further advantages. It is well known that storing specimens in alcohol or formalin is not the best preservative for color pattern, yet it is often the only option. Therefore, most museum specimens and especially old-type specimens (if still available) show significantly less contrast than living or freshly killed specimens, as the original color has faded away. Yet, the investigation of types is still an important aspect in modern science. Applying crossed polarized light filters improves the contrast between parts of the specimen significantly. In our case, the borders of the podomeres of the trunk limbs, which are not apparent under normal light conditions, become marked under crossed polarized light settings (cf. Figure 2(a) versus 2(b)).

2.2.4. Enhancing the Contrast: Use of Fluorescence. Even stronger contrast than that with crossed polarizing filters can be achieved by the application of fluorescence. Fluorescence photography can be done using a camera or a camera in combination with a fluorescence microscope. In the two cases, the optics are much different. In the case of macrofluorescence, that is, with a camera equipped with a macro lens, the contrast stems from significant differences in the color between heavily sclerotized or calcified areas, which appear in our case orange to pink, while membranous areas remain white (Figure 2(c)). The contrast of the borders of the podomeres is significantly higher than with normal whitelight and still better than with crossed polarized light settings.

Using microscopes other than stereo microscopes for documenting the entire specimens is usually limited to very small specimens, as such optical devices have an extremely 
limited depth of field and field of view. With the softwareenhancing tools of image fusion and image stitching, it becomes possible to overcome these shortcomings and document also larger specimens on a microscope. This is especially interesting for applying fluorescence, as the fluorescence light sources on a microscope usually provide a more focused and more uniform light. Since the light produced is monochrome, fluorescence microscopes are generally equipped with $\mathrm{b} / \mathrm{w}$ (monochrome) cameras (see below), which cannot detect color differences. Therefore, fluorescence microscopic images provide differences in brightness between sclerotized/calcified and membranous areas. In the present case, the results are comparable to the macrofluorescence image, based on the recognizability of the podomeres. Yet the fluorescence microscopic image (Figure 2(d), see method below) has a significantly higher resolution than the macrophotographic image (Figure 2(c)), as it is a composite image. This advantage is unfortunately paid by a significantly longer time to produce the image.

While fluorescence has significant advantages for photographing colorless specimens, polarized light settings are superior for photographing freshly killed specimens which still possess their original coloration, as this cannot be well documented with fluorescence imaging. Furthermore, to apply macrophotography, it is necessary to have a completely darkened room, otherwise the white-light from the surroundings will conceal the fluorescence. If such settings are not available, crossed polarized light settings should be chosen. Fluorescence microscopy should only be used to document the entire specimens if they are sufficiently small, or further details are needed. In other cases, the method is simply too time-consuming. For important type specimens, it might still be the method of choice to obtain high-resolution images for having "back-up" information. Such highresolution images could be treated as "virtual specimens".

\subsubsection{Additional 3D Information: Stereo Images. Stereo} images of the entire specimens can also be used to enhance certain morphological details, which would not be visible on a normal macrophotographic image of a not well-contrasted specimen (Figure 3(a)). Spatial arrangements of the legs are well depicted with such a method. The approach to present stereo images to improve the understandability for the reader is also helpful for tomographic data. Different projections can be used for emphasizing different structures in such images. Volume-rendering settings give a good access to structures of the outer surface of the scanned specimen (Figure 3(b), cf. Figure 5(c)). Although in our case the result of the scan is not too highly resolved (see method below), modern micro-CT scanners provide resolutions comparable to overview SEM images (although not comparable in the resolution of higher magnified details, see below). Maximum intensity projections (MIPs) appear more similar to certain light microscopical techniques (Figure 3(c)). It gives the object a more transparent appearance providing access to inner structures, while still showing outer structures. The advantage of these two projection methods is that they can be set up fast, just through adjusting brightness/contrast in

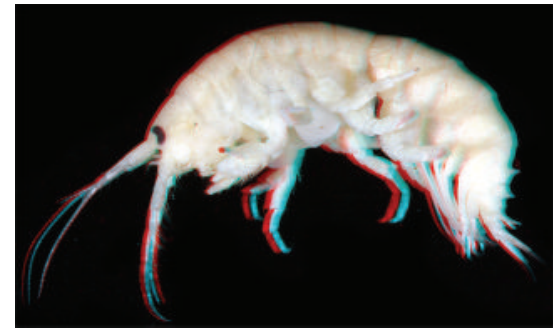

(a)

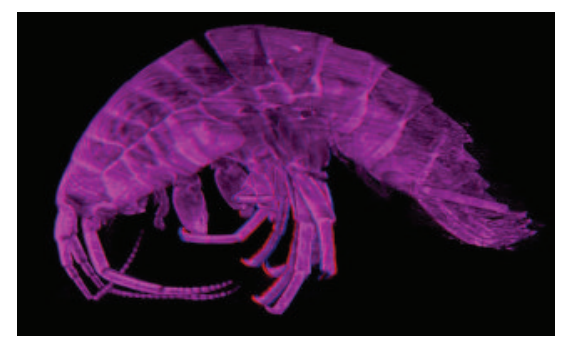

(b)

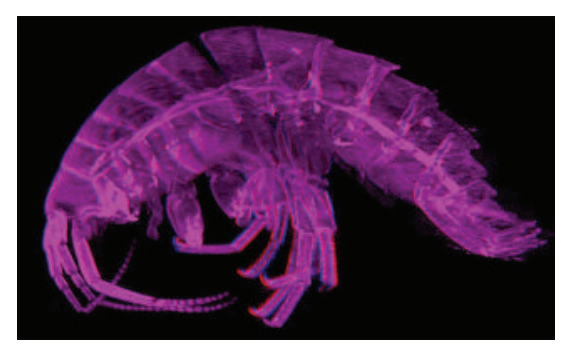

(c)

FIgure 3: Stereo images of entire gammaridean specimens (use red-cyan glasses to view). (a) Red-cyan stereo anaglyph based on a pair of macrographs of Dikerogammarus villosus recorded under slightly different angles. (b)-(c) Images of Gammarus roeselii based on a micro-CT scan. (b) Volume rendering. This presentment emphasizes the outer surface structures. (c) Maximum intensity projection. This presentment enhances the inner structures, as outer surface structures appear transparent.

the projection. More time-consuming is the visualization as a so-called surface model. In this case, it is usually necessary to mark structures by hand, called "segmentation", and this procedure is, therefore, used for emphasizing chosen structures (see below).

\subsection{Documenting Details: Light Microscopy and Comparisons with Alizarin Stainings}

2.3.1. White-Light Microscopy. A mandible of Dikerogammarus haemobaphes was isolated and embedded in glycerol. The distal part of the palp of the mandible was documented in a Zeiss Axioskop using transmitted light under bright field and dark field settings. A Bresser MikroCam directly mounted on the C-mount of the microscope was used for photography. The focal plane was shifted manually, and a stack of frames was recorded. The obtained stack was fused with the software Image Analyzer. Based on the bright field stack, a 3D surface model was generated using the macros of 
Image Analyzer. Stereo images of the "naked" surface model and of a model with the fused image rendered on it were produced by taking screen shots of the model and processing the images in Adobe Photoshop CS3 to red-cyan anaglyphs. The dark field stack was treated following the procedure described by Haug et al. [15] using ImageJ and OsiriX.

\subsubsection{Fluorescence Microscopy. A complete specimen of Di-} kerogammarus villosus (the same specimen as for macrophotographic settings 1-3) was documented using fluorescence microscopy. Only the autofluorescence of the specimen was utilized, and no staining was applied. The specimen was kept in a small petri dish with ethanol and placed on a Zeiss Axio Scope 2 with a $1.25 \times$ objective, equipped with a b/w AxioCam. Several stacks were recorded and processed following the composite image principle (fusion and stitching, see above). The embedded Dikerogammarus haemobaphes mandible used for white-light microscopy was also documented using the Axio Scope 2 with a 10x objective under UV light. In this case, the AxioCam was not used, but an external DCM 510 ocular camera capable of recording live color [9]. Here, in addition, a stack was recorded and fused, but with a sub-optimal result. The distal part of the endopod of the mandible was, therefore, additionally recorded on a Zeiss Observer equipped with a spinning disc and an AxioCam. This stack was three-dimensionally projected with the freely available software OsiriX. From this, a stereo image of a maximum intensity projection (MIP) of this stack was exported.

2.3.3. Alizarin Staining. Another mandible of a specimen of Dikerogammarus haemobaphes was treated after the protocol of Brösing et al. [16] for alizarin staining. This includes macerating the specimen in $10 \% \mathrm{KOH}$ at $100^{\circ} \mathrm{C}$ for one hour, then adding a small amount of alizarin red directly into the solution. After 10-15 minutes, the specimen is removed and rinsed with demineralized water. Afterwards the specimen is stored in $70 \%$ ethanol. Calcified parts manifest red-violet, other structures, especially membranous areas, become transparent. The specimen treated in this way was placed in $70 \%$ ethanol in a petri dish. Reflected light was applied, and a stack of frames was recorded with a Canon macrophoto lens MP-E $65 \mathrm{~mm}$ on a Canon EOS $450 \mathrm{D}$ digital camera.

\subsubsection{Comparing the Results of Light Microscopy and Alizarin} Staining. The possibility to overcome almost any limitation of a restricted depth of field or a limited field of view is a major breakthrough. Although recording stacks is more time-consuming than recording single images, the benefit is overwhelming, especially for high magnification. Here, single images (frames) have an extremely limited depth of field, which is nicely demonstrated by the single image of the mandibular palp tip (Figure 4(a)). Therefore, applying image fusion and also stitching should be seen as mandatory (Figures 4(b) and 4(c)) leading to the complete mandibular palp tip being in focus, including the protruding setae. The technique can be applied to both bright field (Figure 4(b)) and dark field microscopy (Figure 4(c)) and in principle to other contrasting settings. The choice of the proper contrasting method can give access to different structures or at least emphasize different structures. The advantage of bright field microscopy is that the original color of the investigated specimen is evident, depending, of course, if the specimen still has some of its original coloration or if this has already faded. Contrasting settings, such as dark field microscopy, can give access to structures almost invisible in bright field and, furthermore, emphasize thin structures but with the disadvantage of losing the original color information.

Recording stacks has another advantage. The limited depth of field provides information about the threedimensional arrangement of structures, a fact that can be used in different ways to depict three-dimensional arrangements. A fast developing application is the depth from defocus approach (e.g., $[17,18])$. In this case, a surface model is created (Figure $4(\mathrm{~d})$ ), and the fused image based on the stack is rendered onto its surface (Figure 4(e)). The method is usually optimized for conditions in material sciences and industrial applications (e.g., [19]); therefore, there remain some disturbing artifacts for biological specimens, such as the bumpy background (Figures $4(\mathrm{~d})$ and $4(\mathrm{e})$ ). Yet this approach has potential and also demonstrates that it is, in principle, possible to extract $3 \mathrm{D}$ information from a whitelight microscopy stack.

Other approaches were put forward by Kamenz et al. [20], which process the stack in a comparable way to surface models based on CT data sets (see below). The idea to process a stack of light microscopic images following the method of Haug et al. [15] was applied here to the mandibular palp tip (Figure 4(f)). Compared to the depth from defocus approach, color information is lost, although a much more naturally appearing $3 \mathrm{D}$ arrangement of the setae is obtained. It is important to note that all used software for this approach is open access and can be easily applied [15].

Compared to SEM images (cf. Figure 4(g)), most light microscopic images appear less sharp (cf. Figure 4(b)) and also cannot give as good access to the surface structures. A light microscopic technique for accessing surface information can be autofluorescence microscopy (see above for entire specimens). However, autofluorescence is not always coupled to the outer cuticle but can also give access to inner structures. In the present case, we investigated the specimens also using white-light settings. Interestingly, here, the autofluorescence is strongest on the setae. The specimen appears to be close to molting, as under the cuticle a second row of setae is visible. In addition to the rather sharp image detail, this is information that cannot be obtained by either SEM or white-light microscopy. Fluorescence microscopy is, therefore, seen as a technique yielding enormous potential.

Despite this, in cases where the object exhibits strong autofluorescence, this causes scattered light, and the fusion results are often unsatisfying. This is especially true for live color fluorescence microscopy (Figure 4(j)). Unfortunately, most methods to overcome scattered light are coupled to grayscale imaging, such as CLSM, spinning disk (Figure $4(\mathrm{~h})$ ), or apotome. Here, technical enhancements are 

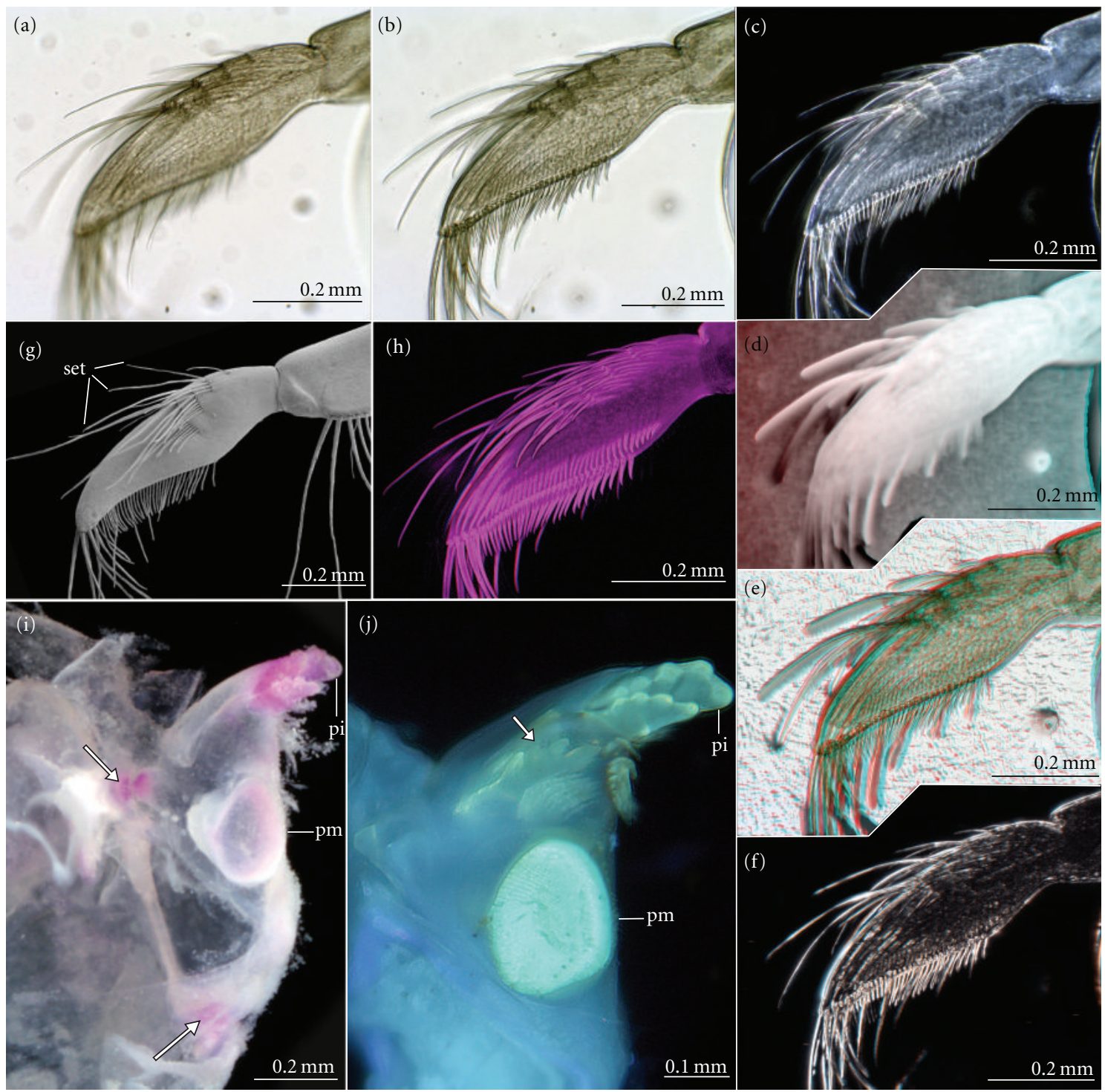

Figure 4: Different microscopical methods applied to structures of the mandible of Dikerogammarus haemobaphes. (a)-(h) Tip of the mandibular palp ((a)-(f) and (h) depict the same specimen). Red-cyan glasses needed to experience the stereo effect in (d)-(f) and (h). (a) A single focal plane of a bright field frame. Note the limited depth of field and the resulting blurring. (b) Fused image based on a stack of 20 frames of different focal planes, including also the frame from (a). (c) Fused image of a stack of 27 dark field frames. (d) Red-cyan stereo anaglyph of a surface model based on stack used for image (b). (e). (b) rendered onto (d). (f) Red-cyan stereo anaglyph based on the stack used for (c), following the procedure of Haug et al. [15]. (g) SEM micrograph of a comparable specimen. (h) Same specimen as in (a)-(f). Red-cyan stereo anaglyph based on an autofluorescence image from an inverted microscope with a spinning disc. The spinning disc suppresses scattered light. Due to the inversion, the other side of the palp is visible (note that the setae seem to come out of the image instead of reaching into it). Image flipped horizontally to enhance comparability. (i) Sagittal cut of the left part of the cephalothorax with left mandible, alizarin staining. Arrows point to the pivots of the joint. (j) Autofluorescence micrograph of a right mandible in median view. Arrow points to new pars incisiva underneath the current cuticle. Abbreviations: set: setae, pi: pars incisiva, pm: pars molaris.

also desirable. However, in this case, the fused images are still significantly better than single images.

\subsubsection{Additional Information from Live Color Fluorescence.} Live color imaging yields another source of data. For understanding the functional morphology of an arthropod, its pattern of sclerotization and, in the case of gammarideans, calcification is of interest. Calcification can well be accessed through staining $\mathrm{KOH}$-macerated specimens with alizarin. We used the protocol provided by Brösing et al. [16] to stain a mandible (Figure 4(i)). Calcified areas appear in violet. In the case of the mandible, these are the tips of the pars incisiva, the pars molaris, as well as the pivot joints of the mandible. Autofluorescence with live color provides a comparable 
image: pars incisiva and pars molaris have a more yellowish color while the remaining cuticle is more bluish under UVfluorescence settings. Autofluorescence may, therefore, be a good alternative for macerating and staining specimens, especially where specimen numbers are limited. Because this is a mandible of the same individual from which the mandibular palp was depicted (Figures $4(\mathrm{a})-4(\mathrm{~h})$ ), the supposed close-to-molt situation can be observed. The new pars incisiva is visible under the old cuticle. Surprisingly, it is quite far away from the old structure. Fluorescence microscopy may be an interesting tool to investigate such close-to-molt situations in detail in the future.

\subsection{Documenting Details: Micro-CT Scanning}

2.4.1. Methods. A critical-point-dried specimen of Gammarus roeselii was documented using a Stratec Fanbeam $\mu$ Scope $\mathrm{X}$-ray micro-CT scanner. The specimen was fixed with a piece of plasticine. The plasticine yielded a weaker contrast than the specimen but had to be virtually removed using the freely available software ImageJ. The produced stack was further processed in OsiriX. The stack was projected as a minimum intensity projection (MIP) and as a volume rendering. From the two projections, Stereo images of lateral views were exported. Two structures, the gut and the endopod of the right gnathopod, were marked by hand in several frames (about every tenth frame). Such a mark is termed a ROI (region of interest). The ROIs missing in the remaining frames were computer-generated using the automatic ROI volume function. The complete process is also termed "segmentation". The segmented structures were highlighted in the MIPs. Additionally, surface models of these ROI volumes were produced and exported as object (.obj) files, an object file being a format for storing a description of the surface of a $3 \mathrm{D}$ object. The surface is described as triangles or higher-degree polygons. Again, a surface model of the entire animal was produced using a relatively high threshold and exported as an .obj file. These .obj files were imported into the freely available 3D software Blender and processed further here (adding color, smoothening, rendering, etc.).

2.4.2. Processing Micro-CT Data. A micro-CT data set can be well used to discuss the possibilities and shortcomings of the principle methodology. While fast projection methods have been discussed above for depicting entire specimens, we focus on the surface model.

For extracting details from a CT data set, it is necessary to go through the process of segmentation (Figures 5(a) and 5(b)). In many cases, this needs to be done by hand, but in well-contrasted data sets, many structures can also be traced via a greyscale threshold. Furthermore, it is usually not necessary to mark the structures in each single frame. Yet, these automatically produced markings should be checked, as in some cases "weird" arrangements are produced with the automated function. Through this process a three-dimensional surface of the marked structure can be generated, which then is usually assigned a certain color.
In the present case, we marked the different podomeres of the endopod of a gnathopod, as well as the gut for orientation (Figure 5(c)). For even better orientation, the outer surface of the specimen was also rendered as a surface model choosing a low threshold giving the surface a skeletonized appearance but providing good reference points (Figure 5(d)). Again it must be mentioned that all the data processing was conducted in OsiriX. The resulting surface model was exported and further treated in the open source 3D software Blender. The fully segmented gnathopod endopod appears "shaved" (Figure 5(e)) compared to an SEM image of the same structure (Figure 5(f)), yet, all podomeres are represented as three-dimensional structures, allowing a proper visualization of the principle geometric shape of all elements. Such 3D models can be further used as a basis for biomechanical investigations.

\subsection{Documenting Details: Scanning Electron Microscopy (SEM, FIB SEM)}

2.5.1. Methods. A Dikerogammarus haemobaphes mandible and the second gnathopod of a Gammarus roeselii specimen were prepared for scanning electron microscopy for further comparisons. To remove debris from the cuticle, the specimens were rinsed in distilled water containing a detergent and sonicated for 20 seconds in a Merck Eurolab ultrasonic cleaner (as recommended by Felgenhauer [21]). Best results were obtained with a solution of a detergent for cleaning dental prostheses. After dehydration in an alcohol series, the specimens were critical-point-dried and sputter-coated with a gold palladium mixture. SEM work was done with a Zeiss DSM 962 scanning electron microscope. A second gnathopod of a specimen of Orchestia cavimana was investigated on a FEI Quanta 3D-FEG focused ion beam SEM (FIB SEM). This machine allows direct microdissection of the specimen while it is in the vacuum chamber of the FIB SEM. A sensillum was dissected in order to have access to the inner architecture of this structure. The digital images obtained from the SEM were trimmed in Adobe Photoshop CS3.

\subsubsection{Access to Minute Details and Internal Structures with} SEM and FIB SEM. Scanning electron microscopy is the method of choice for documenting surface structures when color does not matter. This technique enables magnifications far beyond the possibility of light microscopy, that is, up to 100,000 times magnification. However, in lower magnifications SEM also provides good results, for example, when whole limbs are documented. Although the depth of field of SEM is already much greater compared to light microscopy, it can also be extended by making stacks of frames of different focus planes and fusing them. The problem with SEM might be the accessibility of such machines and the fact that in most cases the specimen has to be dried and sputter-coated. Therefore, SEM should not be applied to rare specimens.

FIB SEM is a new technique that enhances the features of a conventional SEM, with the possibility to dissect minute structures right in the vacuum chamber of a SEM. Not only 


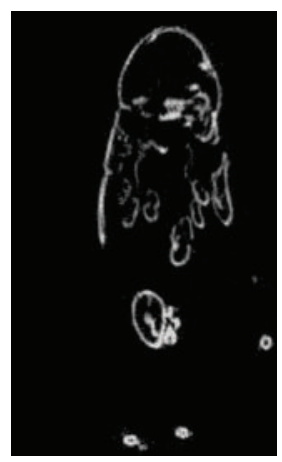

(a)

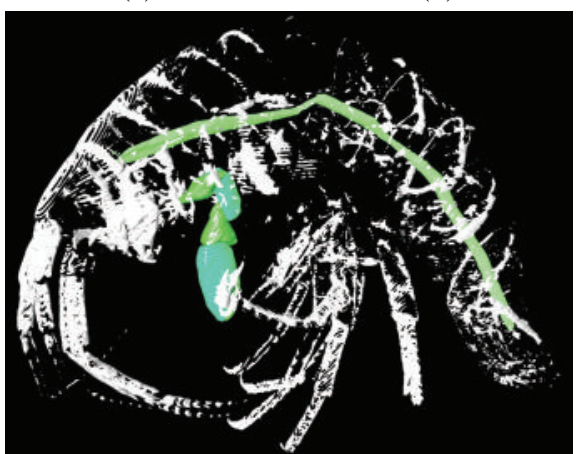

(d)

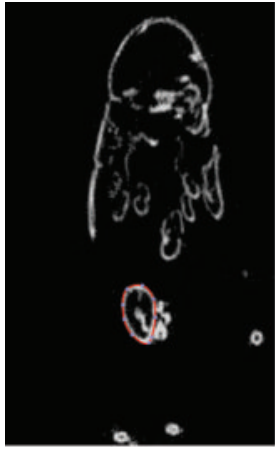

(b)

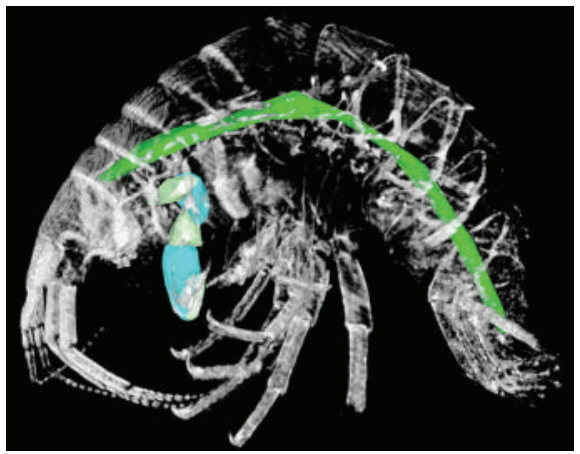

(c)

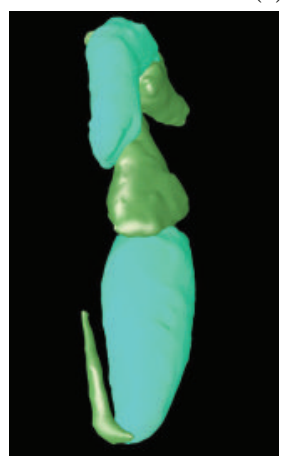

(e)

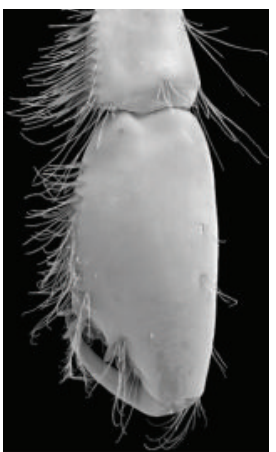

(f)

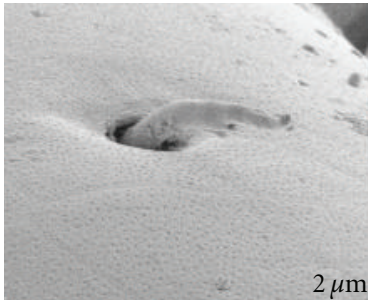

(g)

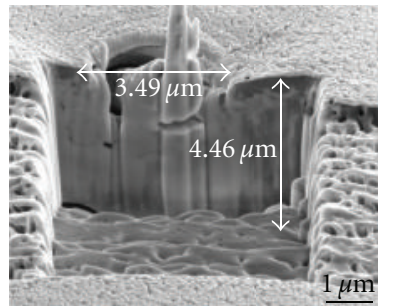

(h)

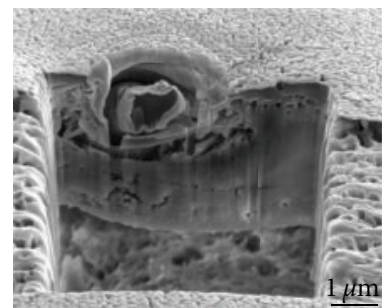

(i)

Figure 5: Micro-CT scans ((a)-(d)) and FIB SEM ((g)-(i)). (a) Single virtual slice of a complete microtomogram of a specimen of Gammarus roeselii. (b) The same image as (a). The outline of the gnathopod element has been marked in OsiriX (red). (c) Volume rendering of the entire microtomogram. The marked structures are the gnathopod and the gut. (d) Comparable image to (c) but now as a surface model. (e) Surface model of the gnathopod after further processing in the 3D-modelling software Blender. (f) The distal part of a right second gnathopod of Gammarus roeselii from posterior as SEM micrograph. (g) A sensillum on the surface of a second gnathopod of Orchestia cavimana. (h) Part of the surface close to the sensillum in (g) milled away. (i) Further milling gives access to the inner structure of the sensillum.

parts of the specimen obscuring the structures of interest can be cut away but also parts of the body surface can be removed to uncover inner structures of the specimen. This is possible through the application of a Gallium ion beam, which can be used to "mill off" structures with an extreme preciseness and fine resolution. In the present case, we applied FIB SEM to investigate the inner structure of a sensillum on the dactylus of a second gnathopod (Figures $5(\mathrm{~g})-5(\mathrm{i}))$. The area in which the milling process was applied was extremely small and could not have been prepared in a comparable way with another method. FIB SEM has a high potential for investigating such minute structures in detail directly after locating them on the specimen. This is regarded as a significant advantage compared to sectioning, which demands to locate the structure before sectioning, then embed and process the specimens and locate the structure of interest again. FIB SEM gives a much more direct access.

\section{Conclusions}

Our overview of different techniques for imaging and documenting gammarideans shows that besides high-tech methods such as FIB SEM and cLSM, there are also facile and inexpensive techniques, which can be applied to gammarideans and other arthropods. We discussed the advantages and disadvantages of the different methods. There is no universal method. The best to apply depends on the individual task. However, testing different methods offers the possibility to reveal new details or simply to get an informative picture of high quality. Apart from that science sometimes might just be beautiful.

\section{Acknowledgments}

This study would not have been possible without dear colleagues providing kind help and access to their facilities. 
Many thanks to the team of the Central Facility for Electron Microscopy, especially Reinhard Weih, the Institute of Analytical and Bioanalytical Chemistry, Focused Ion Beam Center, especially Joel Pikarsky, Boris Mizaikoff, and Christine Kranz, the Institute for Molecular Genetics and Cellular Biology, especially Thomas Gronemeyer, Nils Johnsson, and Judith Müller, Uwe Wolfram at the Institute of Orthopaedic Research and Biomechanics, and Stefan Liebau at the Institute for Anatomy and Cellular Biology, all from the University of Ulm. The authors also thank all people involved in programming freely available software. In the present study, these are particularly GIMP, Microsoft Image Composite Editor, CombineZM, CombineZP, Image Analyzer, Blender, OsiriX, and ImageJ. Special thanks to Calum MacNeil, Isle of Man Government Laboratory at Douglas, Isle of Man for improving the English of the paper. The authors thank one anonymous reviewer for helpful comments. J. T. Haug was kindly funded by the German Research Foundation (DFG) under Wa-754/15-1. This work was part of the project Wa-754/16-1 (DFG).

\section{References}

[1] C. S. Wirkner and L. Prendini, "Comparative morphology of the hemolymph vascular system in scorpions-a survey using corrosion casting, MicroCT, and 3D-reconstruction," Journal of Morphology, vol. 268, no. 5, pp. 401-413, 2007.

[2] A. V. Klaus, V. L. Kulasekera, and V. Schawaroch, "Threedimensional visualization of insect morphology using confocal laser scanning microscopy," Journal of Microscopy, vol. 212, no. 2, pp. 107-121, 2003.

[3] J. Michels, "Confocal laser scanning microscopy: using cuticular autofluorescence for high resolution morphological imaging in small crustaceans," Journal of Microscopy, vol. 227, no. 1, pp. 1-7, 2007.

[4] J. T. Haug, C. Haug, and M. Ehrlich, "First fossil stomatopod larva (Arthropoda: Crustacea) and a new way of documenting Solnhofen fossils (Upper Jurassic, Southern Germany)," Palaeodiversity, vol. 1, pp. 97-110, 2008.

[5] T. A. Hegna, "Photography of soft-bodied crustaceans via drying, whitening, and splicing," Journal of Crustacean Biology, vol. 30, no. 3, pp. 351-356, 2010.

[6] F. Dellaert, S. M. Seitz, C. E. Thorpe, and S. Thrun, "Structure from motion without correspondence," in Proceedings of the IEEE Conference on Computer Vision and Pattern Recognition (CVPR '00), pp. 557-564, June 2000.

[7] C. Haug, J. T. Haug, D. Waloszek, A. Maas, R. Frattigiani, and S. Liebau, "New methods to document fossils from lithographic limestones of Southern Germany and Lebanon," Palaeontologia Electronica, vol. 12, no. 3, article 6T, 2009.

[8] T. Hörnschemeyer, R. G. Beutel, and F. Pasop, "Head structures of Priacma serrata Leconte (Coleoptera, Archostemata) inferred from X-ray tomography," Journal of Morphology, vol. 252, no. 3, pp. 298-314, 2002.

[9] J. T. Haug, C. Haug, V. Kutschera et al., "Autofluorescence imaging, an excellent tool for comparative morphology," Journal of Microscopy, accepted.

[10] D. Platvoet, Z.-E. Hou, S. Li, and G. van der Velde, "Enigmatic pores in females of Dikerogammarus villosus (Sowinsky, 1894) (Amphipoda). Amphipod Pilot Species Project (AMPIS) report 1," Crustaceana, vol. 78, no. 11, pp. 1399-1404, 2006.
[11] D. Platvoet, Y. Song, S. Li, and G. van der Velde, "A lock-on system in precopulae of Dikerogammarus villosus (Sowinsky, 1894), also present in Gammarus pulex pulex (Linnaeus, 1758) (Amphipoda). Amphipod Pilot Species Project (AMPIS) report 2," Crustaceana, vol. 79, no. 8, pp. 993-1003, 2006.

[12] G. Mayer, G. Maier, A. Maas, and D. Waloszek, "Mouthparts of the Ponto-Caspian invader Dikerogammarus villosus (Amphipoda: Pontogammaridae)," Journal of Crustacean Biology, vol. 28, no. 1, pp. 1-15, 2008.

[13] G. Mayer, G. Maier, A. Maas, and D. Waloszek, "Mouthpart morphology of Gammarus roeselii compared to a successful invader, Dikerogammarus villosus (Amphipoda)," Journal of Crustacean Biology, vol. 29, no. 2, pp. 161-174, 2009.

[14] S. Bengtson, "Teasing fossils out of shales with cameras and computers," Palaeontologia Electronica, vol. 3, no. 1, article 4, 2000.

[15] J. T. Haug, C. Haug, A. Maas, S. R. Fayers, N. H. Trewin, and D. Waloszek, "Simple 3D images from fossil and Recent micromaterial using light microscopy," Journal of Microscopy, vol. 233, no. 1, pp. 93-101, 2009.

[16] A. Brösing, S. Richter, and G. Scholtz, "The foregut-ossicle system of Dromia wilsoni, Dromia personata and Lauridromia intermedia (Decapoda, Brachyura, Dromiidae), studied with a new staining method," Arthropod Structure and Development, vol. 30, no. 4, pp. 329-338, 2002.

[17] M. Watanabe, S. K. Nayar, and M. Noguchi, "Real-time computation of depth from defocus," in Three-Dimensional and Unconventional Imaging for Industrial Inspection and Metrology, vol. 2599 of Proceedings of SPIE, pp. 14-25, The International Society for Optical Engineering, Philadelphia, PA, USA, 1995.

[18] S. Chaudhuri and A. Rajagopalan, Depth from Defocus: A Real Aperture Imaging Approach, Springer, Heidelberg, Germany, 1999.

[19] V. P. Namboodiri and S. Chaudhuri, "On defocus, diffusion and depth estimation," Pattern Recognition Letters, vol. 28, no. 3, pp. 311-319, 2007.

[20] C. Kamenz, J. A. Dunlop, G. Scholtz, H. Kerp, and H. Hass, "Microanatomy of Early Devonian book lungs," Biology Letters, vol. 4, no. 2, pp. 212-215, 2008.

[21] B. E. Felgenhauer, "Techniques for preparing crustaceans for scanning electron microscopy," Journal of Crustacean Biology, vol. 7, pp. 71-76, 1987. 

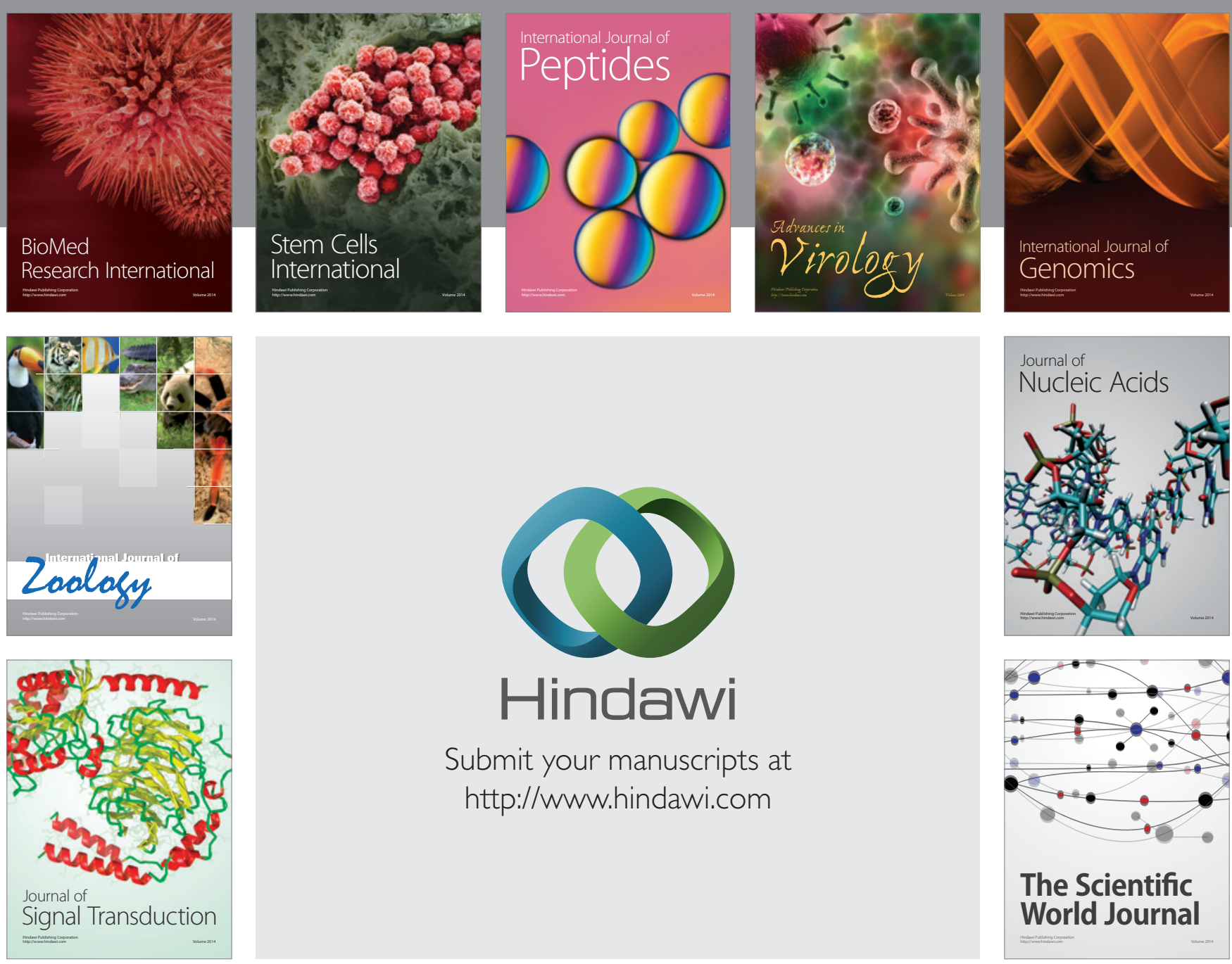

Submit your manuscripts at

http://www.hindawi.com
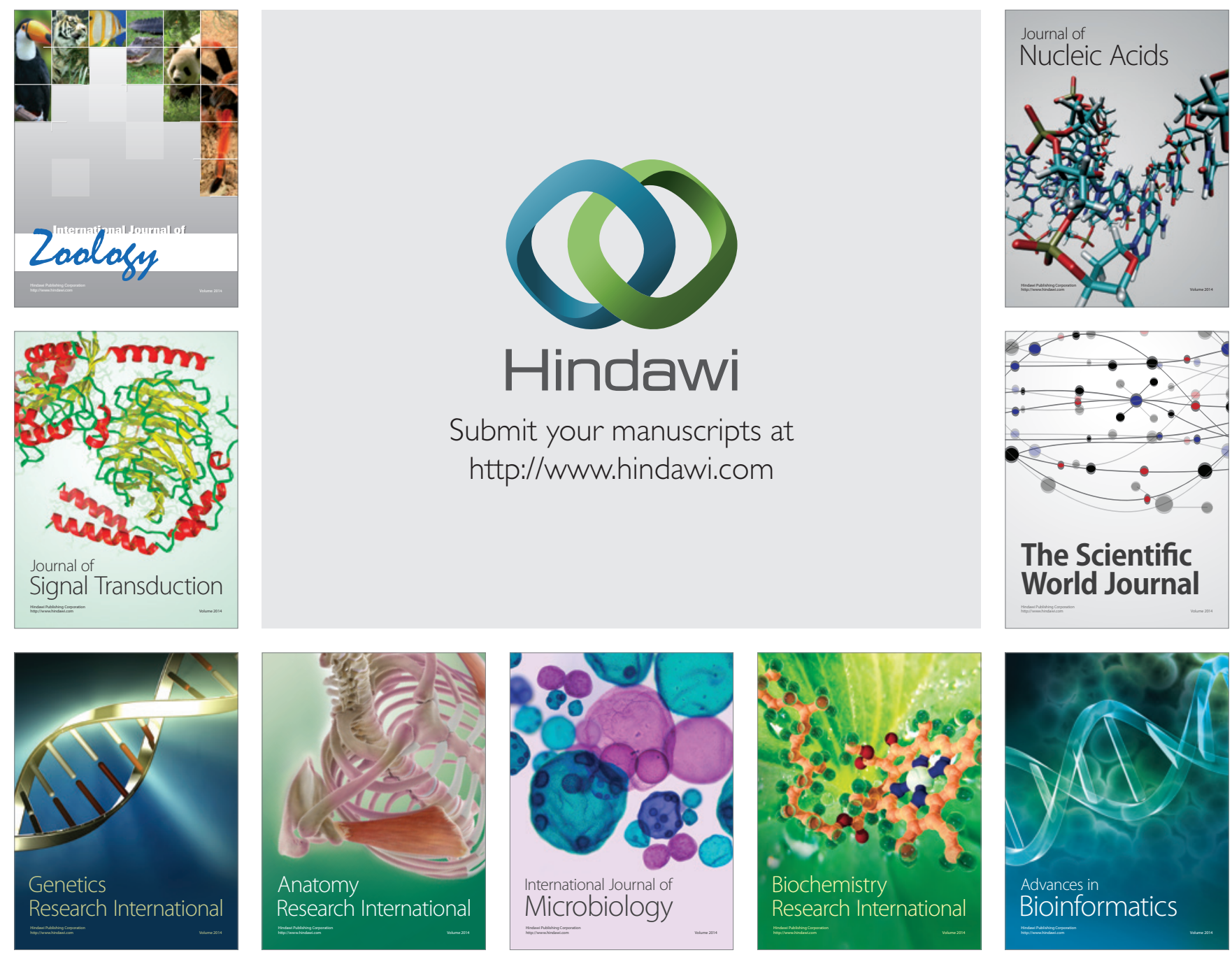

The Scientific World Journal
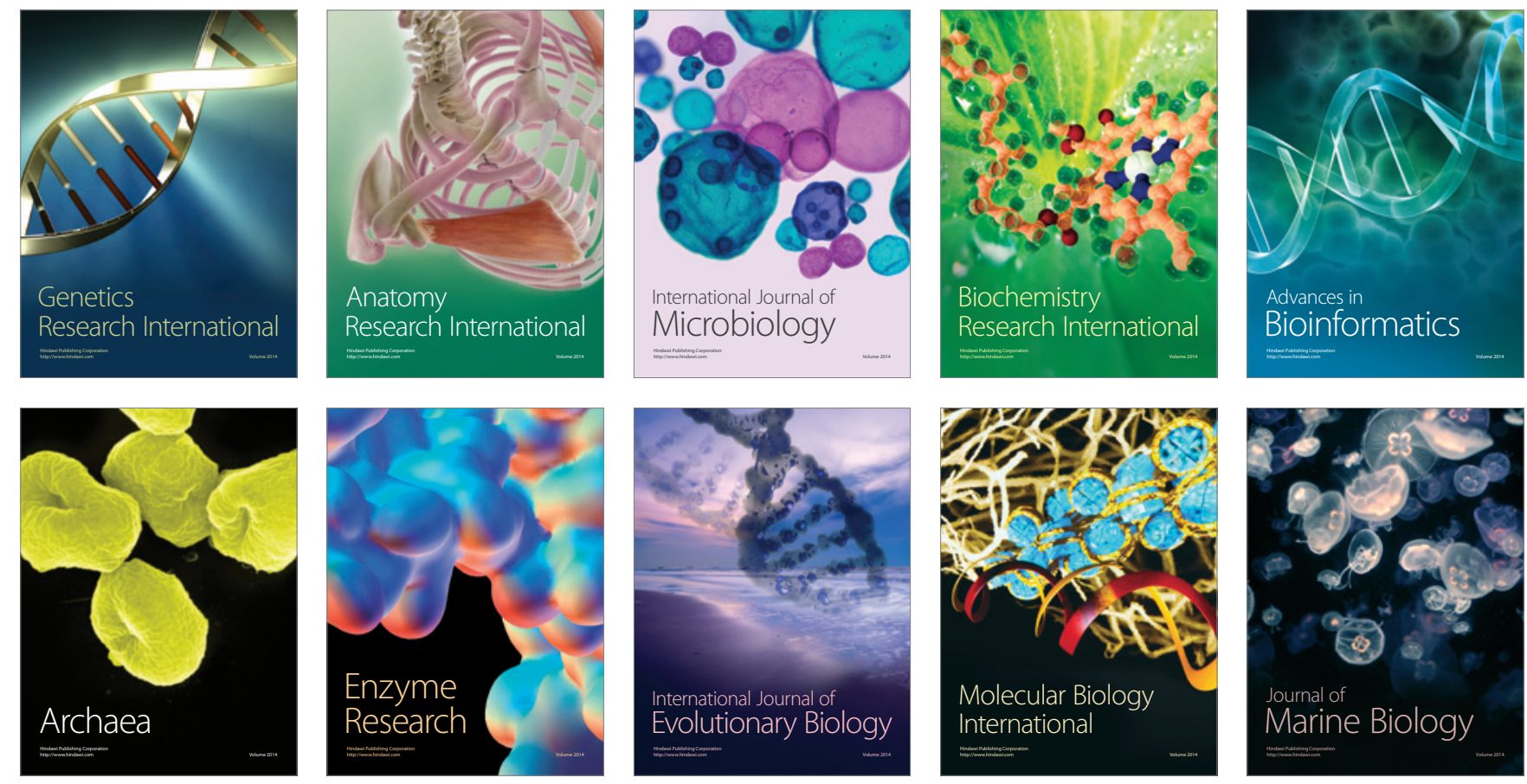